\title{
Formation and Early Evolution of Intermediate Mass Stars
}

\author{
Leonardo Testi \\ INAF - Osservatorio Astrofisico di Arcetri, Firenze, Italy
}

\begin{abstract}
In this paper I discuss our current understanding of the formation of intermediate mass stars and the evolution of their circumstellar material. The results of the search for clusters around Herbig Ae/Be stars are discussed in the context of the possible relationship of the formation of massive stars via coalescence process. Current surveys are not able to offer a definite answer on whether there is a physical relationship between the most massive intermediate mass stars and the presence of clusters. The present day stellar densities in these clusters is too low to allow for frequent stellar collisions, but do not exclude the possibility of coalescence at earlier cluster ages. I review the evidence for and properties of circumstellar disks around Herbig Ae stars, as well as recent observational evidence for dust evolution within these disks. Evidence is presented for the presence of evolved dust in some of these disks, with grain growth to sizes as large as a few centimeters. The status of the search for the more elusive disks around Herbig Be stars is also discussed. Current observations show that disks around Herbig Be stars progenitors may have been common and massive in the protostellar phase, but optically visible Herbig Be stars, albeit very young, are never found to be surrounded by conspicuous dusty disks, suggesting dispersion timescales as short as 1 Myr.
\end{abstract}

\section{Introduction}

In this paper I will review our current understanding of the nature and the formation mechanism of pre-main sequence (PMS) intermediate-mass stars, i.e. stars with masses roughly in the range 2 to $15 \mathrm{M}_{\odot}$, corresponding to main sequence spectral types $\mathrm{A}$ and $\mathrm{B}$. These objects are particularly interesting because they are expected to show the transition from the isolated mode of formation of low-mass T Tauri like stars to the clustered mode of high-mass stars. Intermediate-mass stars with masses close to the lower boundary of the class, are expected to form and evolve in a similar fashion as $\mathrm{T}$ Tauri stars, from the collapse of isolated cloud cores, and to be surrounded by a circumstellar disk that may eventually evolve into a planetary system during the PMS evolution of the star. Stars of larger mass are expected to have short PMS evolution and the most massive members of the intermediate-mass class should not have an optically visible PMS phase at all (Palla \& Stahler 1993). If, as advocated by some authors (Bonnell et al. 1998; Stahler et al. 2000), massive stars form by coalescence of lower mass objects in dense stellar clusters, this different formation 
mechanism may produce observable consequences starting from objects more massive than $\sim 10 \mathrm{M}_{\odot}$, corresponding to intermediate mass stars close to the upper bound of the class.

\section{Herbig Ae/Be stars}

In a search for higher mass counterparts to T Tauri stars, Herbig (1960) reported a list of stellar objects associated with reflection nebulosity, with spectral types $\mathrm{A}$ and $\mathrm{B}$, and with $\mathrm{H} \alpha$ in emission. Since then these objects have been known as Herbig Ae/Be stars. Subsequent studies have confirmed the original suggestion put forward by Herbig, showing that these objects are indeed young pre-main sequence stars more massive than $\mathrm{T}$ Tauri stars. Most of what we know about the formation and evolution of intermediate mass stars comes from detailed studies of the properties of Herbig Ae/Be systems, as will be discussed in the following. Nevertheless, it is important to bear in mind that at present we do not know whether all intermediate-mass stars do go through an "Herbig Ae/Be" evolutionary phase, nor how long this phase lasts (see Herbig 1994). At present, the most extensive catalogue of Herbig Ae/Be stars is that of The et al. (1994), which is, however, by no means complete. In this respect, it is particularly interesting that a survey for PMS stars in the southern hemisphere is being carried out at the Pico dos Dias observatory and that it has already discovered a considerable number of new candidates and confirmed Herbig Ae/Be stars (Gregorio-Hetem et al. 2002).

\section{The onset of clustering around intermediate mass stars}

High mass stars are well known to be associated with clusters and associations, and it has been argued that this association may be linked with their formation mechanism. In fact, coagulation of intermediate mass stars in a young (proto) cluster has been claimed as a possible mechanism to form high mass stars. This mechanism would overcome the difficulty of standard accretion models: they predict that a (proto-)star more massive than $\sim 10 \mathrm{M}_{\odot}$ should be capable of halting accretion with its powerful radiation field and wind (Bonnell et al. 1998; Stahler et al. 2000), although new detailed numerical simulation suggest that the formation of massive stars through disk accretion is a viable possibility (see Yorke this volume). In the coalescence hypothesis, intermediate mass stars are expected to show the transition from the formation mode of low-mass stars in isolation or in loose groups of a few stars per cubic parsec, as observed in nearby star forming regions (e.g. Gomez et al. 1993), to the formation of high mass stars in dense clusters with densities exceeding $10^{4}$ stars per cubic parsec, the nearest example being the Orion Nebula Cluster (Hillenbrand \& Hartmann 1998). Herbig Ae/Be stars are an ideal target for studying the influence of clusters on the formation of massive stars. They are generally not located within large complexes, they suffer low extinction and the burst of star formation that produced them is expected to be over: any possible population of lower mass stars around Herbig $\mathrm{Ae} / \mathrm{Be}$ stars should be easily detectable, especially at near infrared wavelengths.

Guided by these considerations, several searches for clusters around Herbg $\mathrm{Ae} / \mathrm{Be}$ stars have been performed, the more systematic being those of Hillen- 
brand $(1995)$ and Testi et al. $(1997 ; 1998 ; 1999)$. These surveys revealed the presence of small, but rich clusters preferentially around the most massive stars in the observed samples. In particular, while no or loose aggregates are found around Herbig Ae and late type Be stars, early type Be stars are usually found as the most luminous member of well populated clusters. More interestingly, the stellar density of the groups of young stars surrounding Herbig $\mathrm{Ae} / \mathrm{Be}$ stars appears to be a function of the mass of the Herbig star itself, which is always the most luminous member and it is supposed to be the most massive star of the detected cluster. As shown by Testi et al. (1999), the stellar densities in the clusters around Herbig Ae/Be stars fill in the gap between the TTauri stars aggregates in Taurus and the density of the Orion Nebula Cluster. The stellar densities in the clusters around the most massive Herbig Be stars never exceed few times $10^{3}$ stars per cubic parsec, very far from the numbers required by coalescence models $\left(\sim 10^{8}\right.$ stars per cubic parsec) to be efficient in building massive stars via stellar collisions. If coalescence is an important process in the formation of stars in these clusters, it must occur at much earlier times, during a "protocluster" phase when the forming stars are still surrounded by disks and envelopes that may increase by several orders of magnitude the cross section for collision. However, millimeter observations of young protoclusters which are expected to be forming clusters that will eventually look very similar to those surrounding early type Herbig Be stars, such as Serpens (Testi \& Sargent 1998; Testi et al. 2000; Olmi \& Testi 2002) or $\rho$-Oph (Motte et al. 1998), reveal a (proto-)star and prestellar cores density and kinematics that do not seem to favour frequent collisions on a timescale shorter than that expected for the formation of the final stars in the clusters. While it is difficult to rule out definitely the coalescence possibility for the formation of the most massive stars in dense clusters, it is very likely that it cannot play a major role in the formation of intermediate mass stars.

Nevertheless, the observed correlation between cluster richness and density with the mass of the most massive star in the cluster, suggests a possible relationship between the birth of massive stars and the presence of a dense cluster. However, Bonnell \& Clarke (1999) showed that the observed correlation and scatter between the maximum stellar mass and the number of stars in a cluster may be the results of randomly populating according to the stellar IMF clusters extracted from an appropriate size distribution. In this view, the link between massive stars and clusters would not be related to the formation mechanism of massive stars, but simply to the shapes of the stellar IMF and the clusters size distribution.

At this stage the available observational data does not allow us to exclude either the random nor the physical connection between massive stars and clusters. More data have to be collected on a larger sample of massive stars to distinguish between the predictions of these models. These efforts are currently ongoing, by observing a larger sample of Herbig Be and "field" $\mathrm{O}$ stars and by observing intermediate mass protostars (see Testi et al. 2001b; 2001c for preliminary results).

These studies, together with the search and study of massive protostars and protoclusters, are expected to clarify the relationship between massive stars and clusters, and to ascertain whether dense clusters are a necessary ingredient 
for (or a necessary consequence of) the formation of the most massive stars, or whether the association between the two is a mere coincidence.

\section{Disks around Herbig Ae/Be stars}

An alternative approach to study the formation of intermediate mass stars is related to the search and study of circumstellar disks. Circumstellar disks are the landmark of the formation of a stellar system from cloud core collapse, independently of the final mass of the central object. Because of high angular momentum, most of the cloud core material cannot fall directly onto the central protostar and is deposited in a flattened structure rotating about the central object. An optically thick (up to far-infrared wavelengths) disk survives around the formed star, and once this become an optically visible pre-main sequence object, it is observable in the infrared through millimeter wavelength range. While the star evolves to the main sequence, the dust within the disk is expected to coagulate into large grains and eventually planet formation is thought to occur. The optically thick disk is expected to disappear on a timescale of a few million years, possibly a debris disk will form around the central object (Zuckermann 2001). Thus, the study of circumstellar disks around Herbig Ae/Be stars is particularly interesting as it can offer important insights on the formation mechanism of intermediate mass stars and on the possibility of studying the early phases of grain growth and planet formation in these systems.

The presence of disks in Herbig Ae/Be systems have been suggested originally on the basis of their infrared spectral energy distributions (e.g. Hillenbrand et al. 1992). Nevertheless, while the presence of circumstellar material around these stars, in particular dust grains heated by the central star, was clearly indicated by the infrared and millimeter excess emission, the initial hypothesis that it was distributed in a disk-like structure orbiting about the central star have been disputed for a long time. The spectral energy distribution could also be accurately described assuming that the circumstellar material was distributed as a moderately optically thin, spherical shell (e.g. Berrilli et al. 1992; Miroshnichenko et al. 1997). Far-infrared, high angular resolution observations performed with the Kuiper Airborne Observatory showed that the 50 and $100 \mu \mathrm{m}$ emission could be resolved in several of the Herbig $\mathrm{Ae} / \mathrm{Be}$ systems, supporting the conclusion that at least part of the excess emission is due to an extended, optically thin, dusty envelope rather than an optically thick circumstellar disk (Natta et al. 1993; Di Francesco et al. 1998).

\subsection{Disks in Ae systems}

The disk/envelope debate was finally resolved, at least for Ae systems, thanks to millimeter wavelength interferometric observations that resolved the disk structure and kinematics (Mannings \& Sargent 1997ab; 2000; Di Francesco et al. 1997). In fact, it is now clear that the properties of disks around Herbig Ae stars are very similar to those of disks around T Tauri stars (Natta et al. 2000). Standard flared disk models, appropriately modifed to take into account the photospheric parameters of the central star, can explain all the features observed in the Ae systems, including the polarization pattern observed during UX Ori 
variables minima, the silicate emission at $10 \mu \mathrm{m}$ and the $3 \mu \mathrm{m}$ excess (Natta et al. 2001; Natta \& Whitney 2001; Dullemond et al. 2001).

Given the higher luminosity of the central object, the disks in Ae systems are hotter and more luminous than those in T Tauri systems. It is thus easier to study disk evolution and the initial stages of the planetary formation process. A few Herbig Ae systems show a millimeter wavelength spectral energy distribution that can be fit as power law, $\mathrm{F}_{\nu} \sim \nu^{\alpha_{\mathrm{mm}}}$, with a remarkably low value of the index, $\alpha_{\mathrm{mm}} \sim 2$. Such a low value of the spectral index at mm wavelengths, where the dust emission is expected to be optically thin, suggests that the dust opacity coefficient do not depend on wavelength, i.e. that the size of the grains is much larger than the wavelength at which the observations are performed (Natta et al. 2001). To test this possibility, Testi et al. (2001a; 2003) and Natta et al. (2003) have performed an extensive $\mathrm{cm}$ and $\mathrm{mm}$ wavelength interferometric study of a sample of eight Ae systems. VLA $3.6 \mathrm{~cm}$ observations were used to evaluate the possible contribution of gas emission to the millimeter fluxes, while $1.3-2.6 \mathrm{~mm}$ PdBI and $7 \mathrm{~mm}$ VLA observations were used to constrain the dust thermal emission. The observed spectral energy distributions show a relatively low power law exponent at millimeter wavelength, with spectra indices between 1.3 and $7 \mathrm{~mm}$ in the range 2-3. Considering also published data at other wavelengths, these results can be interpreted as due to: i) truncated, optically thick (at $\mathrm{mm}$ wavelengths) disks with standard dust particles, or ii) average size disks composed by dust with a dust opacity coefficent very different from the interstellar medium. At millimeter wavelengths, the dust opacity coefficient can be approximated as $\mathrm{k}_{\nu} \propto \mathrm{k}_{1.3 m m} \nu^{\beta}$, the value of $\beta$ for standard interstellar grain mixtures is in the range 1.5-2.0; our results suggest that if the disks are optically thin at millimeter wavelengths the dust emission is well explained with $\beta \leq 0.6$. This value for the power law exponent of the dust opacity coefficient is expected only for dust distributions with very large grains, with radii up to several centimeters (Miyake \& Nakagawa 1993; Natta et al. 2003).

VLA C-array $7 \mathrm{~mm}$ high angular resolution $\left(0.5^{\prime \prime}\right)$ observations of two objects in our sample (CQ Tau and HD 163296) have shown that the disks are resolved with radii of 100-300 $\mathrm{AU}$, ruling out, at least for these two systems, the possibility of very small optically thick disks and confirming the grain growth hypothesis (Testi et al. 2003; Natta et al. 2003). The study of these systems is particularly interesting as grain growth is the first step in the planet formation process. The old Herbig Ae/Be disks may represent an intermediate stage between the molecular disks around intermediate mass protostars (e.g. Beltrán et al. 2002) and young Herbig Ae stars (e.g. Mannings et al. 1997a), and the more evolved debris disks around older A-type stars, such as HR4796A (Koerner et al. 1998) or $\beta$-Pic (Smith \& Terrile 1984), where planet formation is thought to have already occurred.

\subsection{Disks in Be systems}

The evidence for the presence of circumstellar disks in the more massive Be systems is much more elusive than in Ae systems. Be stars are on average much more distant from the Sun than Ae systems, thus it is more difficult to detect and resolve the structure of the possible disks using interferometers. The limited millimeter interferometric observations available to date show that, if present, 
the amount of dust in the disks around Herbig Be stars has to be lower than what expected from the extrapolation of lower mass systems (Natta et al. 2000). Nevertheless, there are indications that gaseous disks may exists in Be systems from $\mathrm{H} \alpha$ spectropolarimetry (Vink et al. 2003), the interpretation of these data is not unique, and the lack of millimeter emission from the dusty or molecular components of the presumed disks is puzzling. Very recently, deep PdBI and VLA millimeter interferometric observations of a sample of Herbig Be stars, have shown the possible detection of disks in two sources, the inferred disk masses are however very low (Fuente et al. 2003). It is clear that the study of these systems require more sensitive millimeter continuum and molecular line observations.

Is the lack of massive disks around Herbig Be stars due to an evolutionary effect or is it somehow related to the formation mechanism of these stars? Even if Be stars are on average younger than Ae stars, they interact more strongly with the surrounding material, and the lack of conspicuous disks may be due to a rapid clearing of the circumstellar material by the hot central object. Fuente et al. $(1998 ; 2002)$ have studied in detail the molecular environment around Herbig $\mathrm{Ae} / \mathrm{Be}$ stars and have shown that Be stars do disperse the parent molecular clumps on a much shorter timescale. It is however still unclear whether they can also disperse with the same efficiency dense circumstellar disks, as detailed photoevaporation calculations suggest timescales longer than the typical age of Herbig Be stars (Hollenbach et al. 2000).

From an observational point of view, there have been many attempt to detect disks in younger intermediate mass (proto-)stars. These studies are complicated by the difficult task of finding nearby, bona fide, intermediate mass protostars, that can then be observed with millimeter interferometers to asses the presence and properties of circumstellar disks. So far the best examples are represented by the sources IRAS20126+4104 (Cesaroni et al. 1997; 1999) and G192.16-3.82 (Shepherd et al. 1999; 2001). Both objects are characterized by a bolometric luminosity typical of early B stars, power large bipolar outflows and collimated jets, and show evidence for rotating disk-like structures close to the central object.

These initial findigs suggest that massive circumstellar disks are present at the early stages of the formation of intermediate mass stars, but evolve very rapidly and may dissipate on timescales shorter than $1 \mathrm{Myr}$. A more detailed study of the disk frequency and evolution, as well as its relation with accretion onto the central object, is needed.

\section{References}

Beltrán, M.T., Girart, J.M., Estarella, R., Ho, P.T.P., Palau, A. 2002, ApJ, 573, 246

Berrilli, F., Corciulo, G., Ingrosso, G., Lorenzetti, D., Nisini, B., Strafella, F. 1992, ApJ, 398, 254

Bonnell, I.A. \& Clarke, C.J. 1999, MNRAS, 309, 461

Bonnell, I.A., Bate, M.R., Zinnecker, H. 1998, MNRAS, 298, 93

Cesaroni, R., Felli, M., Testi, L., Walmsley, C.M., Olmi, L. 1997, A\&A, 325, 725 
Cesaroni, R., Felli, M., Jenness, T., Neri, R., Olmi, L., Robberto, M., Testi, L., Walmsley, C.M. 1999, A\&A, 345, 949

Dullemond, C.P., Dominik, C., Natta, A. 2001, ApJ, 560, 957

di Francesco, J., Evans, N.J.II, Harvey, P.M., Mundy, L.G., Butner, H.M. 1998, ApJ, 509, 324

di Francesco, J., Evans, N.J.II, Harvey, P.M., Mundy, L.G., Guilloteau, S., Chandler, C.J. 1997, ApJ, 482, 433

Fuente, A., Martin-Pintado, J., Bachiller, R., Neri, R., Palla, F. 1998, A\&A, 334,253

Fuente, A., Martin-Pintado, J., Bachiller, R., Rodriguez-Franco, A., Palla, F. 2002, A\&A, 387, 977

Fuente, A., Rodríguez-Franco, A., Testi, L., Natta, A., Bachiller, R., Neri, R. 2003, ApJ, in press

Gregorio-Hetem, J., et al. 2003, in preparation

Herbig, G.H. 1960, ApJS, 4, 337

Herbig, G.H. 1994, in "The Nature and Evolutionary Status of Herbig Ae/Be Stars", P.S. The, M.R. Perez \& E.P.J. Van den Heuvel eds., ASP Conf. Series, Vol. 62 , p. 3

Hillenbrand, L.A., Hartmann, L.W. 1998, ApJ, 492, 540

Hillenbrand, L.A., Strom, S.E., Vrba, F.J., Keene, J. 1992, ApJ, 397, 613

Hillenbrand, L.A. 1995, PhD Thesis, University of Massachusetts

Hollenbach, D.J., Yorke, H.W., Johnstone, D. 2000, in "Protostars \& Planets IV", V. Mannings, A. Boss \& S.S. Russell eds., (Tucson: Univ. of Arizona Press), p. 401

Mannings, V.G., Koerner, D.W., Sargent A.I. 1997a, Nature, 388, 555

Mannings, V.G. \& Sargent A.I. 1997b, ApJ, 490, 792

Mannings, V.G. \& Sargent A.I. 2000, ApJ, 529, 391

Miroshnichenko, A., Ivezic, Z., Elitzur, M. 1997, ApJ, 475, L44

Miyake, K. \& Nakagawa, Y. 1993, Icarus, 106, 20

Moscadelli, L., Cesaroni, R., Rioja, M.J. 2000, A\&A, 360, 663

Motte, F., André, Ph., Neri, R. 1998, A\&A, 336, 150

Natta, A., Grinin, V., Mannings, V. 2000, in Protostars $\&$ Planets IV, V. Mannings, A. Boss \& S.S. Russell eds., (Tucson: Univ. of Arizona Press), p. 559

Natta, A., Palla, F., Butner, H.M., Evans, N.J.II, Harvey, P.M. 1993, ApJ, 406, 674

Natta, A., Prusti, T., Neri, R., Wooden, D., Grinin, V.P., Mannings, V. 2001, ApJ, 371, 186

Natta, A., Testi, L., Shepherd, D.S., Wilner, D.J. 2003, A\&A, submitted

Natta, A., Whitney, B. 2000, A\&A, 364, 633

Olmi, L. \& Testi, L. 2002, A\&A, 392, 1053

Palla, F. \& Stahler, S. 1993, ApJ, 418, 414

Shepherd, D.S., Claussen, M.J., Kurtz, S.E. 2001, Science, 292, 1513 
Shepherd, D.S., Kurtz, S.E. 1999, ApJ, 523, 690

Smith, B. A., \& Terrile, R. J. 1984, Science, 226, 4681

Stahler, S.W., Palla, F., Ho, P.T.P. 2000, in "Protostars \& Planets IV", V. Mannings, A. Boss \& S.S. Russell eds., (Tucson: Univ. of Arizona Press), p. 327

Testi, L., Natta, A., Shepherd, D.S., Wilner, D.J. 2001a, ApJ, 554, 1087

Testi, L., Natta, A., Shepherd, D.S., Wilner, D.J. 2003, A\&A, 403, 323

Testi, L., Palla, F., Natta, A., Prusti, T., Maltagliati, S. 1997, A\&A, 320, 159

Testi, L., Palla, F., Natta, A. 1998, A\&AS, 133, 81

Testi, L., Palla, F., Natta, A. 1999, A\&A, 342, 515

Testi, L., Palla, F., Natta, A. 2001b, in From Darkness to Light: Origin and Evolution of Young Stellar Clusters, T. Montmerle \& Ph. André eds, ASP Conf. Series V. 243, p. 377

Testi, L. \& Sargent, A.I. 1998, ApJ, 508, L91

Testi, L., Sargent, A.I., Olmi, L., Onello J. 2000, ApJ, 540, L53

Testi, L., Vanzi, L., Massi, F. 2001c, The ESO Messenger, 103, 28

The, P.S., de Winter, D., Perez, M.R. 1994, A\&AS, 104, 315

Vink, J., Drew, J., Harries, T. \& Oudmaijer, R., 2003, in "Galactic Star Formation across the Stellar Mass Spectrum", ASP Conf. Series V. 287, p. 319

Zuckermann, B. 2001, ARAA, 39, 549 